OPEN ACCESS

Edited by:

Joshua Thomas Butcher,

Oklahoma State University,

United States

Reviewed by:

Michael Sturek,

Indiana University Bloomington

School of Medicine, United States

Ellen Gillis,

Augusta University Medical College of

Georgia, United States

*Correspondence:

Guo-Gang Zhang

zhangguogang@csu.edu.cn

Rui-Zheng Shi

xyshiruizheng@csu.edu.cn

Specialty section:

This article was submitted to

Vascular Physiology,

a section of the journal

Frontiers in Physiology

Received: 13 September 2020 Accepted: 24 February 2021

Published: 30 March 2021

Citation

Wang $Q-C$, Wang $Z-Y, X u Q$,

Li R-B, Zhang G-G and Shi R-Z

(2021) Exploring the Role of Epicardial

Adipose Tissue in Coronary Artery

Disease From the Difference of Gene

Expression

Front. Physiol. 12:605811. doi: 10.3389/fphys.2021.605811

\section{Exploring the Role of Epicardial Adipose Tissue in Coronary Artery Disease From the Difference of Gene Expression}

\author{
Qian-Chen Wang ${ }^{1}$, Zhen-Yu Wang ${ }^{2}$, Qian X $u^{3}$, Ruo-Bing Li', Guo-Gang Zhang ${ }^{1 *}$ and \\ Rui-Zheng Shi* \\ 1 Department of Cardiovascular Medicine, Xiangya Hospital, Central South University, Changsha, China, ${ }^{2}$ Department \\ of Cardiovascular Medicine, The Second Xiangya Hospital, Central South University, Changsha, China, ${ }^{3}$ Department \\ of Cardiovascular Surgery, Xiangya Hospital, Central South University, Changsha, China
}

Objectives: Epicardial adipose tissue (EAT) is closely adjacent to the coronary arteries and myocardium, its role as an endocrine organ to affect the pathophysiological processes of the coronary arteries and myocardium has been increasingly recognized. However, the specific gene expression profiles of EAT in coronary artery disease (CAD) has not been well characterized. Our aim was to investigate the role of EAT in CAD at the gene level.

Methods: Here, we compared the histological and gene expression difference of EAT between $C A D$ and non-CAD. We investigated the gene expression profiles in the EAT of patients with CAD through the high-throughput RNA sequencing. We performed bioinformatics analysis such as functional enrichment analysis and protein-protein interaction network construction to obtain and verify the hub differentially expressed genes (DEGs) in the EAT of CAD.

Results: Our results showed that the size of epicardial adipocytes in the CAD group was larger than in the control group. Our findings on the EAT gene expression profiles of CAD showed a total of 747 DEGs (fold change $>2, p$ value $<0.05$ ). The enrichment analysis of DEGs showed that more pro-inflammatory and immunological genes and pathways were involved in CAD. Ten hub DEGs (GNG3, MCHR1, BDKRB1, MCHR2, CXCL8, CXCR5, CCR8, CCL4L1, TAS2R10, and TAS2R41) were identified.

Conclusion: Epicardial adipose tissue in CAD shows unique gene expression profiles and may act as key regulators in the CAD pathological process.

Keywords: epicardial adipose tissue, coronary artery disease, gene expression profiles, bioinformatics analysis, mRNA

\section{INTRODUCTION}

Obesity, hypertension, type 2 diabetes, hyperlipidemia, and other pathogenic factors are all associated with coronary artery disease (Gensini et al., 1998; Zengin et al., 2015; Messerli et al., 2019). Recent studies have found that in addition to the accumulation of peripheral fat, the increase in local epicardial fat is also one of the important risk factors for coronary artery disease (CAD) 
(Franssens et al., 2017). In addition to storing fat, adipose tissue has been increasingly recognized as an endocrine organ, particularly because it is related to glucose and lipid homeostasis (Chong et al., 2017; Wong et al., 2017; Gruzdeva et al., 2019). Excessive calorie intake will trigger chronic inflammatory changes in adipose tissue (Sekimoto et al., 2015), and the link between adipose tissue inflammation and CAD has been gradually attracting attention.

Adipose tissue mainly includes two types, white adipose tissue (WAT) and brown adipose tissue (BAT). In terms of morphology and distribution, WAT containing unilocular lipid droplets is the main body fat, which contains less cytoplasm and mitochondria. BAT containing polylocular lipid droplets is mainly distributed in the inside of the scapula or around the kidney. Its mitochondria express abundant uncoupling protein 1 (UCP1), and are rich in blood vessels and innervation. Cold and $\beta 3$ receptor stimulation can trigger the yielding of UCP1-positive cells in WAT, which are similar in shape to BAT adipocytes, with multilocular lipid droplets and abundant mitochondria, and are named as "browning" adipocytes (Alipoor et al., 2020; Reinisch et al., 2020). Under physiological conditions, Epicardial adipose tissue (EAT) belongs to WAT, but under pathological conditions, UCP1 expression increases in EAT which then presents "browning" changes (Chechi et al., 2017). Clinical studies have found that EAT deposition is positively correlated with increased metabolic abnormalities and CAD (Milanese et al., 2020).

Epicardial adipose tissue covers almost all coronary arteries anatomically (Iacobellis, 2015). By wrapping around and directly contacting the coronary arteries without fascia separation, EAT is able to affect the pathophysiological process of coronary arteries. EAT is mainly located in the atrioventricular and ventricular sulcus. It is regarded as a unique adipose depot with unique metabolic characteristics, which has a higher ability to absorb and release fatty acid (FFA) than other visceral fats and is an effective source of FFA that fuels the energyintensive myocardial tissue (Kankaanpää et al., 2006; Pezeshkian and Mahtabipour, 2013). Many studies have shown that CAD patients have more expressions and pro-inflammatory cytokines production in the EAT than in the subcutaneous adipose tissue (SAT) (Vacca et al., 2016; Mráz et al., 2019). The EAT in CAD patients expresses pro-inflammatory adipokines and is accompanied by the infiltration of various immune cells (Nomura et al., 2020). However, most studies only compare the difference between EAT and SAT in an individual with CAD and lack the comparison with the EAT in subjects with normal coronary arteries. Therefore, it is difficult to estimate the scale of the EAT pro-inflammatory effect on coronary arteries in CAD.

In this study, we first directly compared the gene expression in the EAT of subjects with and without CAD through RNA sequencing (RNA-Seq), and obtained the gene expression profiles of EAT in CAD, and then performed bioinformatics analysis such as functional enrichment analysis and protein-protein interaction (PPI) network construction, etc., and obtained and verified differentially expressed genes with research value.

\section{MATERIALS AND METHODS}

\section{Study Population}

The study subjects were 11 patients with CAD who underwent coronary artery bypass surgery and 11 patients without CAD who underwent valve replacement in the Department of Cardiovascular Surgery of Xiangya Hospital of Central South University. The selective and exclusive criteria were as follows: (1) CAD group were patients with CAD who underwent coronary artery bypass surgery (confirmed by coronary angiography); (2) Control group (CTRL group) were patients with valvular disease who matched the age, gender, and body mass index (BMI) of the CAD group and underwent valve replacement with no evidence of CAD syndrome and negative findings in coronary angiography; (3) The main exclusive criteria included a previous PCI history, history of diabetes mellitus, previous thoracotomy history, tumor, severe infection, and autoimmune diseases. We selected five pairs from the two groups matched in age, gender, BMI, and abdominal circumference (AC), and RNAseq was performed on the selected pairs. All patients underwent a preoperative risk assessment and signed informed consent forms. This study was approved by the Ethics Committee of Xiangya Hospital of Central South University. Trial registration: Chinese Clinical Trial Registry, No. ChiCTR1900024782.

\section{Sample Collection}

Before starting the extracorporeal circulation, the samples of EAT were taken from uninjured areas near the anterior descending coronary artery or along the atrioventricular sulcus near the right coronary artery. The samples were quickly frozen with liquid nitrogen for molecular biology experiments and stored at $-80^{\circ} \mathrm{C}$.

\section{Histology}

The EAT samples were fixed in 4\% paraformaldehyde, embedded in paraffin, and cut into $4 \mu \mathrm{m}$ thick slices. The size of the adipocyte was determined by calculating the Feret's diameter of the H\&E stained slices (Lin et al., 2013). For each slice, we used the Image-Pro 6.2 software to check five fields of view and calculated the average.

\section{RNA Sequencing}

Total RNA was extracted from the samples using TRIzol. RNase $\mathrm{H}$ method was used to remove rRNA. After RNA fragmentation, cDNA synthesis, A-Tailing Mix, and adapters were added to establish a library. An Agilent 2100 Bioanalyzer (Agilent DNA 1000 Reagents) was used to detect the inserted fragments of the library, and HiSeq4000 (BGI-Shenzhen, China) was used to sequence the library qualified for detection.

\section{Functional Enrichment Analysis of DEGs}

The database for annotation, visualization, and integrated discovery (DAVID 6.8 ${ }^{1}$ ) (Huang da et al., 2009) was used to analyze the functional enrichment of key differentially expressed genes (DEGs) in the GO Terms and KEGG Pathways. The threshold was $p<0.05$.

\footnotetext{
${ }^{1}$ http://david.ncifcrf.gov
} 


\section{PPI Network Construction and Analysis}

Studying the interaction between the encoded proteins will help us to discover the core regulatory genes. Thus, in order to explore the DEGs with a research value, a search tool (STRING 10.5²) (Szklarczyk et al., 2019) was used to establish a PPI network, and Cytoscape (Shannon et al., 2003) was used to plot the network. Interactions with a score $>0.4$ were set as the cutoff point. The most important modules in the PPI network were identified using MCODE in Cytoscape, which clustered a given network through topology. The threshold was set as: MCODE scores $>5$, degree cutoff $=2$, node score cut-off $=0.2$, max depth $=100$, and $k$-score $=2$. Subsequently, the maximal clique centrality (MCC) algorithm of CytoHubba was used to explore the hub genes of the PPI network.

\section{Real-Time Quantitative PCR}

Total RNA was extracted from the samples using TRIzol (Takara) according to the manufacturer's instruction. We used the cDNA synthesis kit (Takara) to conduct reverse transcription. Real-time quantitative PCR (RT-qPCR) was performed by the FastStart Universal SYBR Green Master (ROX) (Takara). $2^{-\Delta \Delta C t}$ relative quantification method was used for relative expression. $\beta$-actin was used as the internal reference (Sangon Biotech). The primer sequences were as following: UCP1 forward CGACGTCCAGTGTTATTAGGTA, UCP1 reverse GTAGAGTTTCATCCGCCCTTC, GNG3 forward CGGTGAACAGCACTATGAGTAT, GNG3 reverse TCACAGTA AGTCATCAGGTCTG, MCHR1 forward CGCTTGGTCC TGTCGGTGAAG, MCHR1 reverse GCCTTTGCTTTCTG TCCTCTCCTC, BDKRB1 forward CTTTTTGTCCTGTTGG TCTTCC, BDKRB1 reverse CTGATGAACAAATTGGCCTTGA, MCHR2 forward AACCTGGCTGTGGCTGATTTGG, MCHR2 reverse GGGATGTGATGATGGTGCAGAGAG, CXCL8 forward AACTGAGAGTGATTGAGAGTGG, CXCL8 reverse ATGA ATTCTCAGCCCTCTTCAA, CXCR5 forward CGGCAG ACACGCAGTTCCAC, CXCR5 reverse ACGGCAAAGGGCAA GATGAAGAC, CCR8 forward TGGCCCTGTCTGACCT GCTTT, CCR8 reverse GGCATAAGTCAGCTGTTGGCT, CCL4L1 forward CTCAGTATCAGCACAGGACACAGC, CCL 4L1 reverse AGAGACAGGACAGTCACGCAGAG, TAS2R10 forward AGTGTTTGGGGTTTTGGGGAATGG, TAS2R10 reverse AGCCGGTGAGAATAAAGCCAATCG, TAS2R41 forward ACTCAGCCACCTTCTGGTTTTGC, and TAS2R41 reverse ATCAGGACAGAGCCCAACAGGAG.

\section{Statistical Analysis}

The statistical analysis was performed by SPSS25.0 (IBM). All values were expressed as mean \pm SD. Statistically significant differences were assessed by ANOVA and Chi-squared test for comparisons between two groups. Two-tailed $p$ value $<0.05$ was considered statistically significant.

\footnotetext{
${ }^{2}$ http://string-db.org
}

TABLE 1 | Patient characteristics.

\begin{tabular}{lccc}
\hline & CTRL $(\boldsymbol{n}=\mathbf{1 1})$ & CAD $(\boldsymbol{n}=\mathbf{1 1})$ & $\boldsymbol{p}$ values \\
\hline Age (years) & $56.0 \pm 8.0$ & $58.8 \pm 7.0$ & 0.392 \\
Sex, male (\%) & $6(54.5)$ & $5(45.5)$ & 0.748 \\
BMI (Kg/m²) & $22.5 \pm 2.4$ & $23.8 \pm 2.1$ & 0.197 \\
AC (cm) & $86.1 \pm 10.3$ & $87.2 \pm 6.6$ & 0.779 \\
Complications & & & \\
Diabetes mellitus (\%) & $0(0.0)$ & $0(0.0)$ & 1.000 \\
Hypertension (\%) & $3(27.2)$ & $5(45.5)$ & 0.478 \\
CAD & & & \\
1-Vessel disease & $0(0.0)$ & $1(9.1)$ & \\
2-Vessel disease & $0(0.0)$ & $4(36.4)$ & \\
3-Vessel disease & $0(0.0)$ & $6(54.5)$ & \\
Gensini score & $3.6 \pm 1.4$ & $88.4 \pm 43.7$ & $<0.001$ \\
Laboratory measurements & & & \\
WBC (×10 $/$ L) & $5.9 \pm 2.7$ & $6.5 \pm 1.4$ & 0.513 \\
TG (mmol/L) & $1.6(0.6-2.6)$ & $1.6(1.2-2.0)$ & 0.997 \\
TC (mmol/L) & $4.2 \pm 0.8$ & $4.6 \pm 1.7$ & 0.411 \\
LDL-c (mmol/L) & $2.6 \pm 0.5$ & $2.9 \pm 1.3$ & 0.472 \\
Left heart function & & & \\
EF (\%) & $55.6 \pm 9.9$ & $58.0 \pm 12.6$ & 0.636 \\
\hline
\end{tabular}

Data are presented as mean $\pm S D$ or the number (\%) of patients.

$B M I$, body mass index; AC, abdominal circumference; WBC, white blood cell; TGs, triglycerides; TC, total cholesterol; LDL-c, low-density lipoprotein cholesterol; EF, ejection fraction.

\section{RESULTS}

\section{Study Population}

The clinical characteristics of the study subjects are listed in Table 1. There was no significant difference in age, gender, body mass index (BMI), abdominal circumference (AC), comorbidities, and plasma lipid levels between the two groups. The average age of the control subjects was $56.0 \pm 8.0$ years and the CAD subjects was $58.8 \pm 7.0$ years $(p=0.392)$. Therefore, our research groups were well matched except for the presence of CAD.

\section{Comparison of EAT in Histology}

The EAT samples of both groups were mainly unilocular white adipocytes (Figure 1A). The sizes of EAT adipocytes in the CAD group were significantly larger than those in the control group $\left(126.59 \pm 23.27 \mu \mathrm{m}^{2}\right.$ and $\left.66.45 \pm 14.90 \mu \mathrm{m}^{2}, p<0.001\right)$ (Figure 1B). Then, we compared the "browning" of fat in each group by detecting the expression of UCP1 (Figure 1C) and found that the level UCP1 was not different in the two groups $(p>0.05)$.

\section{EAT Gene Expression Profiles in CAD}

In order to compare the difference of gene expression in EAT between the CAD and non-CAD groups, we used the highthroughput RNA sequencing. The thresholds of differentially expressed genes (DEGs) were fold change $>2$ and adjusted $p$ value $<0.05$. In total, there were 747 DEGs, of which, 301 were significantly up-regulated in the CAD group and 446 genes were significantly down-regulated (Figure 2A). Enrichment 

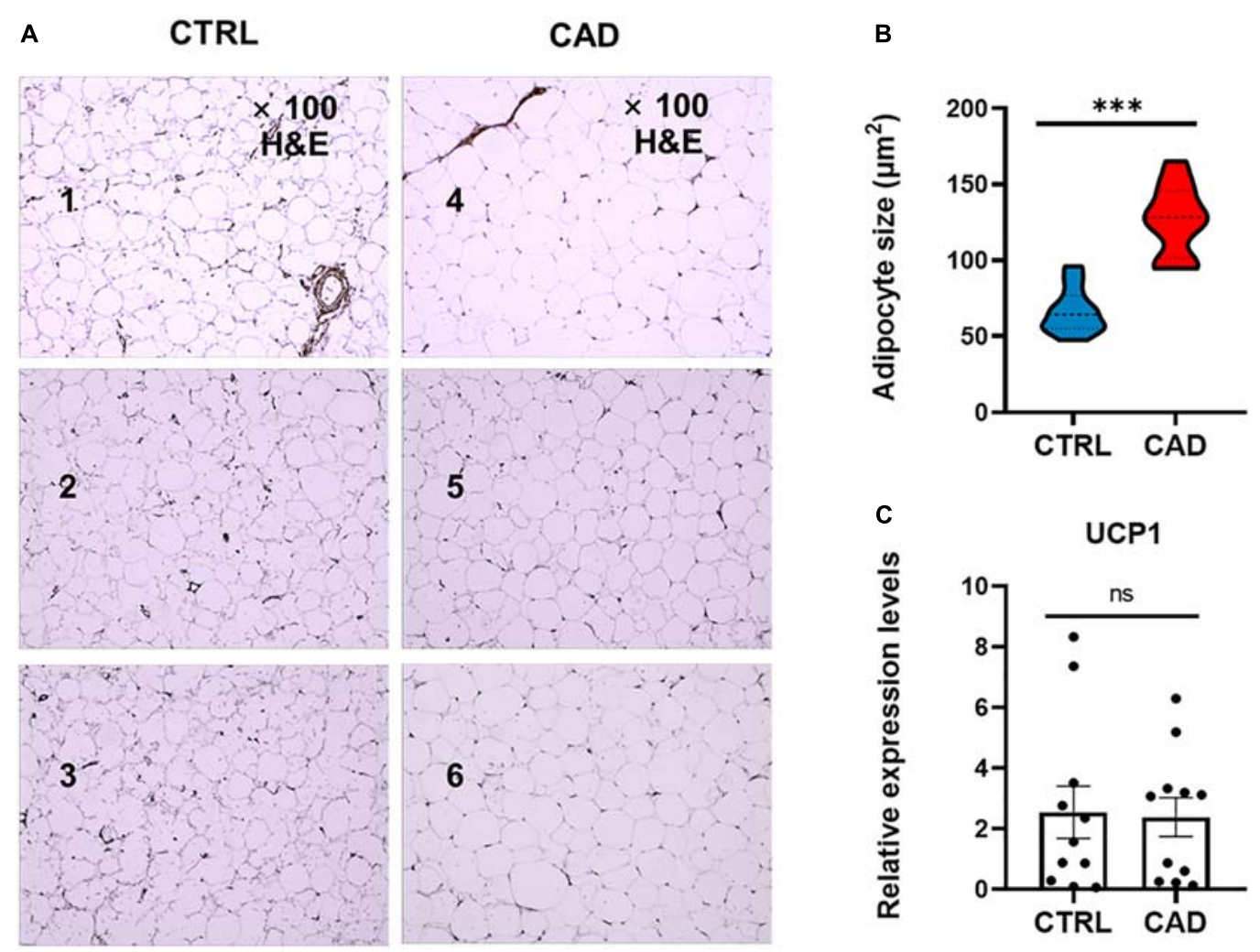

FIGURE 1 | Comparison of EAT in histology. (A) H\&E staining of epicardial adipose tissue (EAT) in the control and coronary artery disease (CAD) groups (100x magnification). Numbers 1-3 were EAT samples of three control subjects, while numbers 4-6 were EAT samples of three CAD patients. (B) The mean adipocyte size was larger in CAD group ( $\left.{ }^{\star \star *} p<0.001\right)$. (C) Expression levels of uncoupling protein 1 (UCP1) in the CTRL and CAD group (ns, $p>0.05$ ).

analysis of 301 up-regulated differential genes showed that the KEGG pathways were significantly enriched in cytokine-cytokine receptor interaction, jak-STAT signaling pathway, nicotine addiction, chemokine signaling pathway, hematopoietic cell lineage, steroid hormone biosynthesis, butanoate metabolism, and neuroactive ligand-receptor interaction (Figure 2B). The top eight GO analysis results were listed in Table 2 . The biological processes $(\mathrm{BP})$ of $\mathrm{GO}$ analysis showed that genes were mainly enriched in epidermis development, cytoskeleton organization, cellular calcium ion homeostasis, chemokine-mediated signaling pathway, chemotaxis, neutrophil chemotaxis, steroid metabolic process, and lymphocyte chemotaxis.

\section{PPI Network Construction and Hub Genes Verification}

To further identify the key genes with research value, 301 upregulated DEGs were submitted to the STRING database to predict the interaction between proteins. DEGs' PPI network was constructed with a comprehensive score greater than 0.4 (Figure 3A), and the most important module was obtained using MCODE in Cytoscape. The first module included 14 nodes and 67 edges; the second module included eight nodes and 28 edges; and the third module included six nodes and 15 edges. The hub genes were selected from the PPI network using the maximal clique centrality (MCC) algorithm of CytoHubba
(Figure 3B). The top 10 hub genes identified by MCC were GNG3, MCHR1, BDKRB1, MCHR2, CXCL8, CXCR5, CCR8, CCL4L1, TAS2R10, and TAS2R41. RT-qPCR was performed using the total RNA extracted from 11 pairs of EAT to verify the expression levels of the hub genes. The results of the RTqPCR showed significant changes in the expression levels of hub genes such as GNG3, MCHR1, BDKRB1, CCR8, and TAS2R41 in Figure 4 ( $p$ value $<0.05)$.

\section{DISCUSSION}

Epicardial adipose tissue directly contacts with the coronary arteries without fascia isolation, which provides an important anatomical basis for the interaction between them. EAT can be used as a target for drugs targeting adipose tissue. New hypoglycemic drugs, such as glucagon-like peptide-1 receptor (GLP-1R) agonists and glucose-sodium co-transporter 2 inhibitors (SGLT2i) (Bouchi et al., 2017; Iacobellis et al., 2017), and lipid-lowering drugs such as atorvastatin (Raggi et al., 2019), have been shown to directly target the EAT and reduce its volume. GLP-1R's regulation of EAT promotes fatty acid $\beta$-oxidation and white-to-brown adipocyte differentiation, promoting favorable metabolic changes. Clinical studies have shown that EAT is remarkably correlated with the presence 

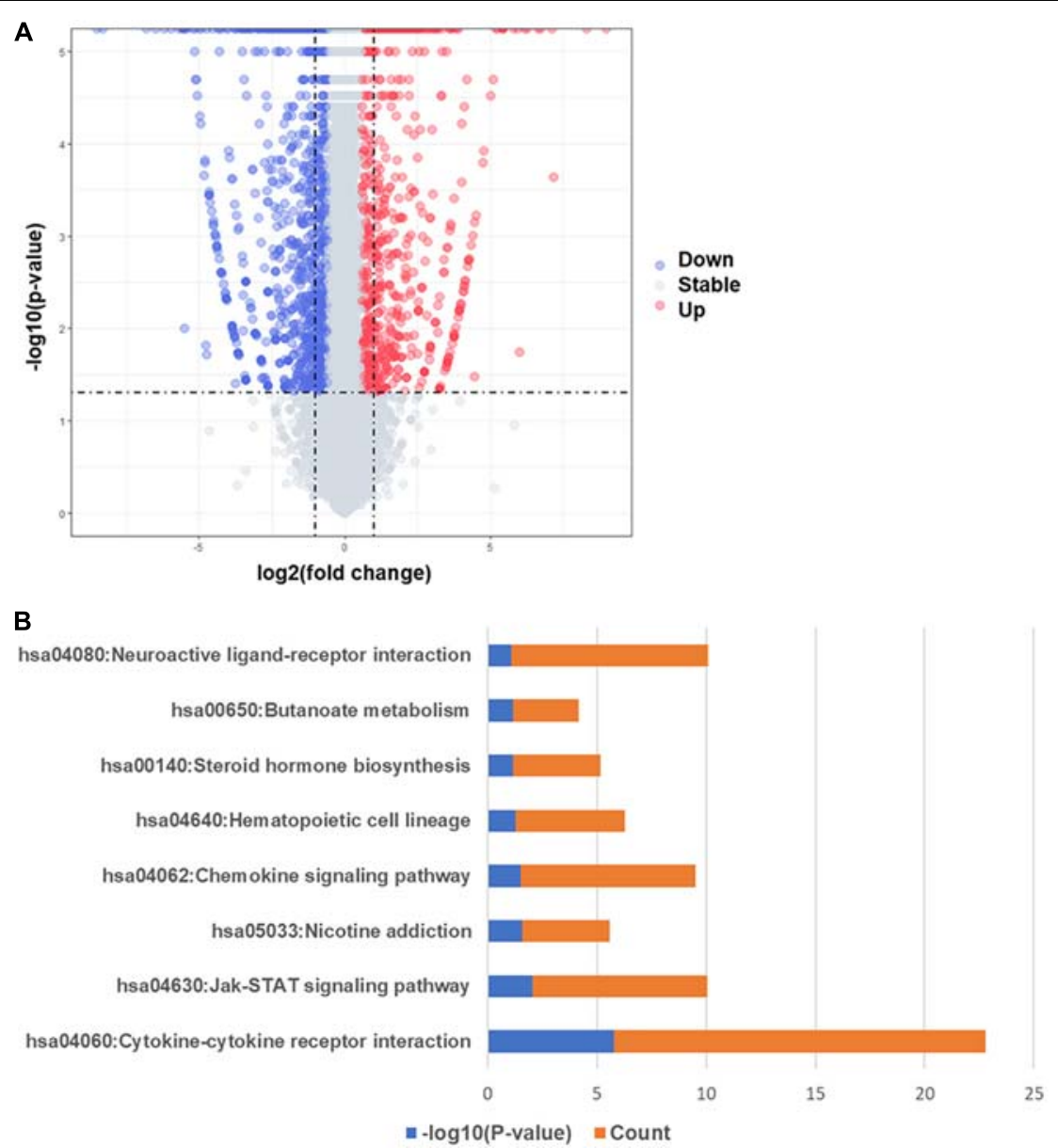

FIGURE 2 | Differentially expressed genes and KEGG pathway enrichment analysis. (A) Volcano map of differentially expressed genes, red spots represent up-regulated genes and blue spots represent down-regulated genes. (B) KEGG pathways significantly associated with the upregulated genes.

of CAD (Le Jemtel et al., 2019; Liu et al., 2019; Nappi et al., 2019). Previous studies have focused on comparing the difference between EAT and SAT. Different from previous studies, we directly compared the differences in EAT gene expression profiles between CAD and non-CAD subjects, providing novel information to describe the genomic characteristics of EAT in healthy and diseased subjects. Then, we provided evidence that, compared with the control group, EAT in CAD was characterized by enhanced inflammatory genes, metabolic remodeling, and fat remodeling. These results might help us discover the key genes and therapeutic targets in $\mathrm{CAD}$, which might provide a more theoretical basis for exploring the pathogenesis of CAD.

Our research confirmed the findings of some previous studies and suggested some potentially novel discoveries. Our results showed that the size of epicardial adipocytes in the CAD group was larger than the control group, which was consistent with the results of previous studies (Vianello et al., 2016). However, we found that the "browning" marker UCP1 was not significantly different in the two groups. Our findings of the EAT gene expression profiles of CAD showed a total of 747 DEGs, of which, 301 DEGs were up-regulated and 446 DEGs were down-regulated. It was notable that the enrichment analysis of DEGs showed that more pro-inflammatory and immunological genes and pathways were involved in CAD, including chemokinemediated signaling pathway, chemotaxis, neutrophil chemotaxis, lymphocyte chemotaxis, chemokine activity, cytokine activity, cytokine-cytokine receptor interaction, and chemokine signaling pathway. This suggested that inflammatory factors and immune cell activation might play an important role in the regulation of CAD. We constructed the DEG's PPI network in which three key modules and 10 central genes were explored, including GNG3, MCHR1, BDKRB1, MCHR2, CXCL8, CXCR5, CCR8, CCL4L1, TAS2R10, and TAS2R41. In addition, we used RT-qPCR to further verify the results.

A meta-analysis focusing on the transcriptome assessment of EAT in CAD patients confirmed the activation of inflammatory, immune, and metabolic pathways in CAD-EAT, and highlighted interleukin-6 (IL-6) and tumor protein p53 (TP53) as core genes (Maghbooli and Hossein-Nezhad, 2015). McKenney et al. (2014) demonstrated in a porcine model that the resection of local EAT can prevent CAD progression. These results suggest that EAT dysfunction may lead to changes in 
TABLE 2 | The significant GO enriched analysis of differentially expressed genes in CAD.

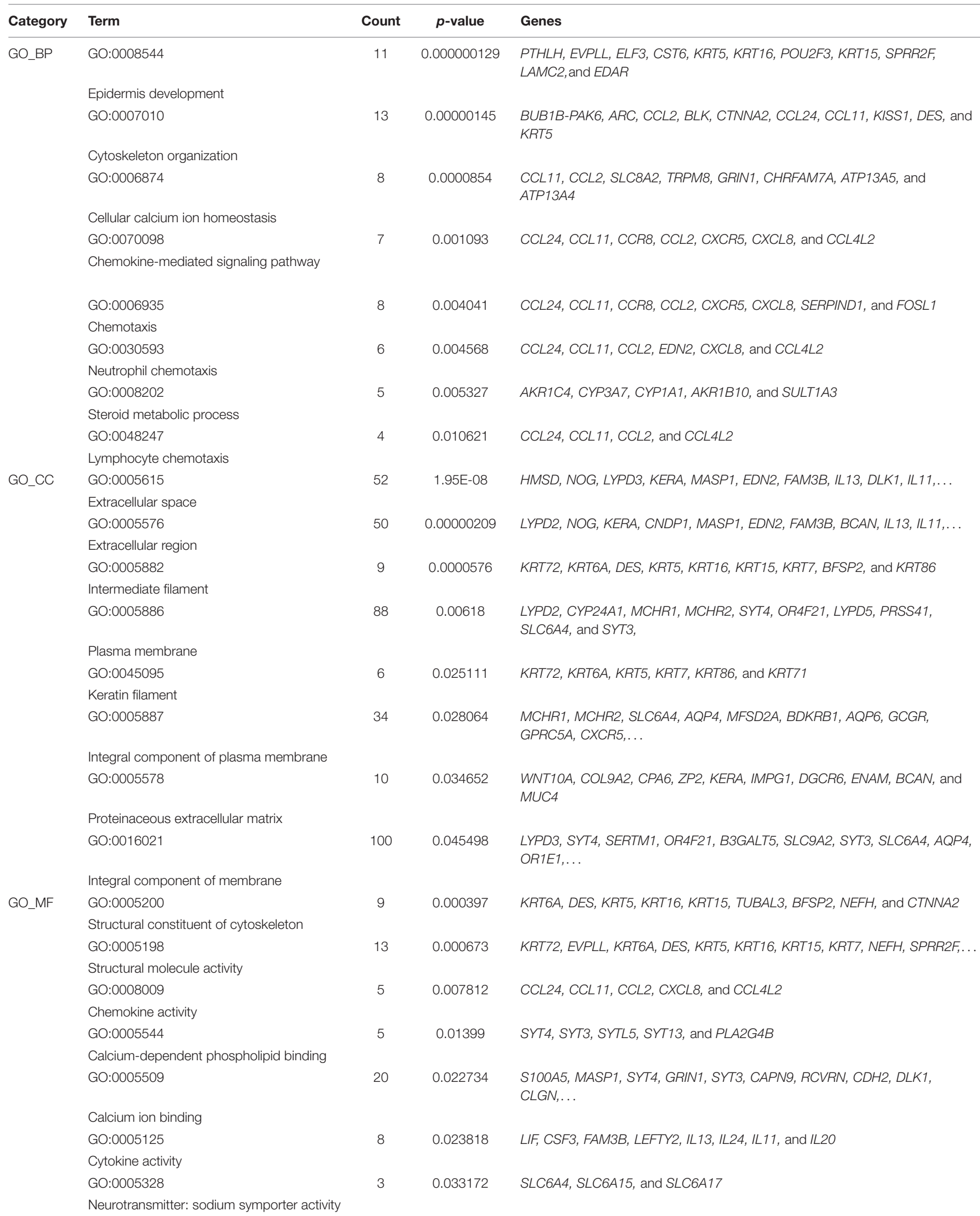




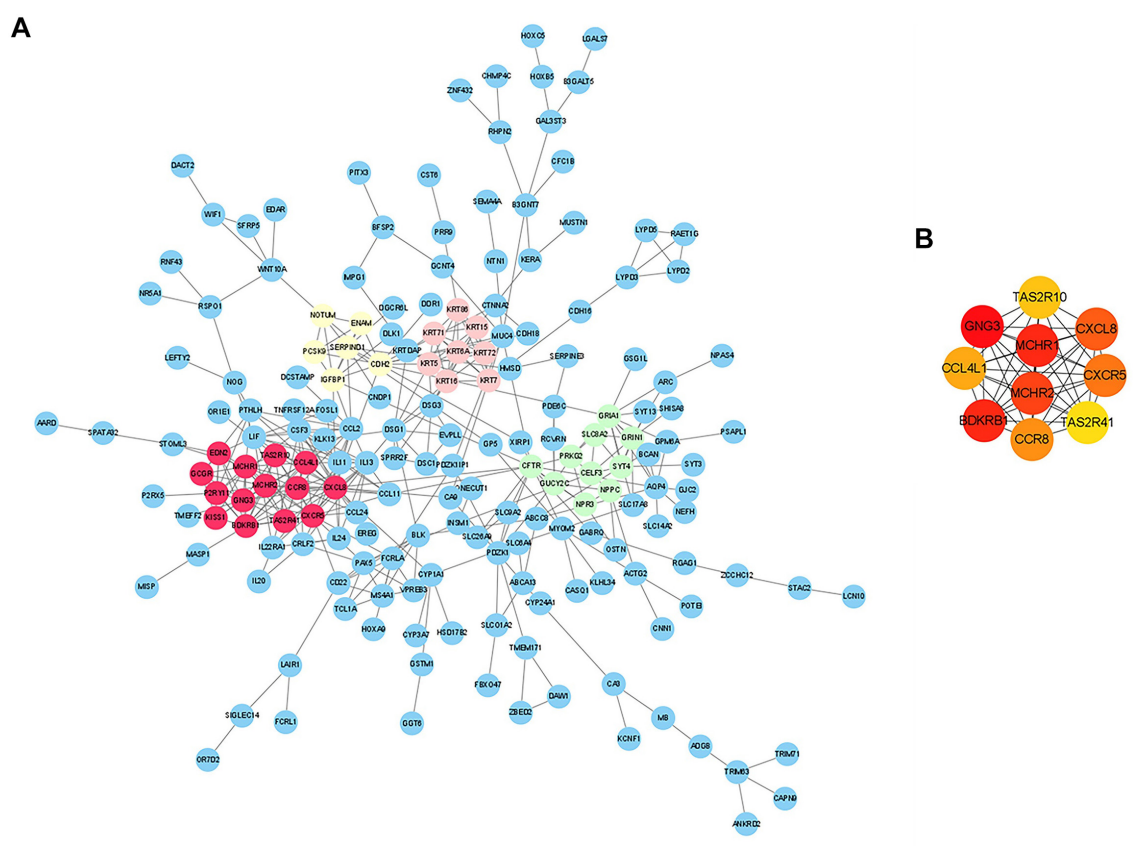

FIGURE 3 | Analysis of protein-protein interaction (PPI) network of CAD-related differentially expressed genes (DEGs) in EAT. (A) Edges represented protein-protein associations which were meant to be specific and meaningful. The colored nodes (red, green, pink, and yellow) represented the top four modules in the PPI network. (B) Top 10 hub genes identified by CytoHubba in Cytoscape.

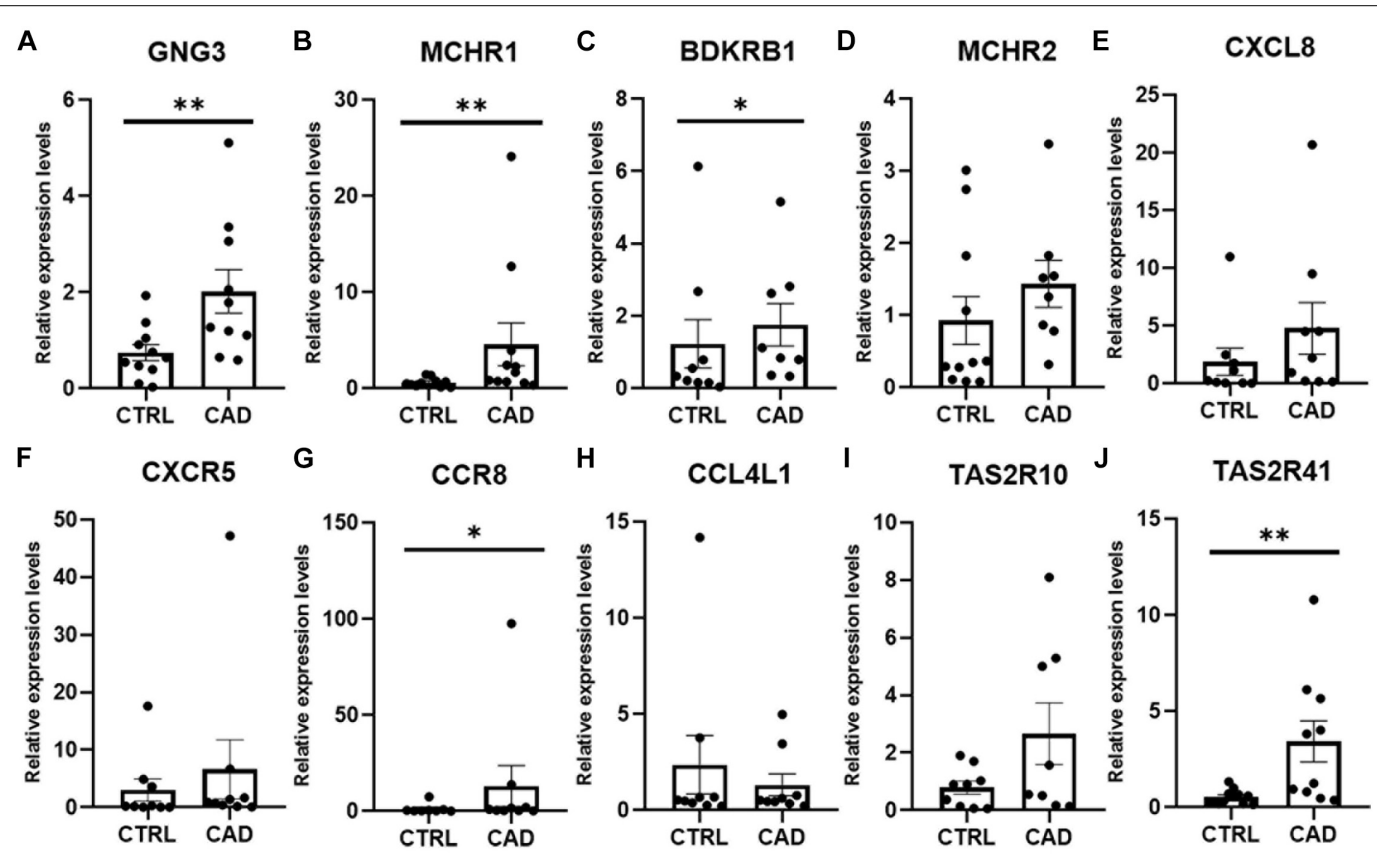

FIGURE 4 | Real-time quantitative PCR (RT-qPCR) verification of 10 hub genes in EAT of CAD. (A) GNG3 expression was significantly increased in the CAD group. (B) MCHR1 expression was significantly higher in the CAD group. (C) BDKRB1 expression was greater in the CAD group. (D) MCHR2 was increased in the CAD group, although the difference was not statistically significant. (E) CXCL8 expression was not statistically different between the two groups. (F) CXCR5 expression was higher in the CAD group, although the difference was not statistically significant. (G) CCR8 expression was significantly higher in the CAD group. (H) CCL4L1 expression was not different in the two groups. (I) TAS2R10 expression was increased in the CAD group, although the difference was not statistically significant. (J) TAS2R41 expression was significantly increased in EAT of CAD. ${ }^{*} p<0.05,{ }^{*} p<0.01$. In each column, error bars show the mean and standard deviation per group. 
inflammation and metabolic microenvironment, thereby affecting vascular homeostasis, and may trigger coronary atherosclerosis. We found that the salient features of EAT in CAD include an enhanced communication between the inflammatory cells and chemokine signaling (CXCL8, CXCR5, CCR8, and CCL4L1). Studies have confirmed that many CC and CXC chemokines are involved in cardiovascular diseases (Frangogiannis and Entman, 2005; Frangogiannis, 2014). Cytokines in the EAT of CAD are also gradually attracting attention. Immunocyte like monocytes and neutrophils infiltrate the intima and activate endothelial cells, which induce the differentiation of monocytes into macrophages and formation of foam cells. CXC chemokines like CXCL8 was reported to control neutrophil infiltration (Dyer et al., 2014) and CXCR5 + T cells was found to contribute to inflammatory reactions in CAD (Ding et al., 2017). CCR8 can recruit IL-5 $+\mathrm{T}(\mathrm{H}) 2$ cells (Islam et al., 2011) and act as a driver of atherosclerosis (Gast et al., 2019). CCL4L1 may play a role in aortic aneurysm (Gäbel et al., 2017) though its involvement in CAD has yet to be clarified. Overall, our findings indicate that the immunological function of CXCL8, CXCR5, CCR8, and CCL4L1 in the pathogenesis of EAT in CAD may be the focus of future investigations.

The other six hub genes had been rarely studied in the field of cardiovascular research. GNG3 has not been reported in CAD. However, Schwindinger et al. (2004) reported that the mice which were lacking GNG3 had a significantly reduced weight. Balber et al. (2019) reported that MCHR1 expression was increased in BAT, and the use of MCHR1 antagonists in rodents was able to reduce adipogenesis. It is well known that obesity is a risk factor of $\mathrm{CAD}$ and the findings of these genes may suggest that they may be indirectly involved in CAD pathogenesis. The discovery of the melanin-concentrating hormone $(\mathrm{MCH})$ and its receptors (MCHR1 and $M C H R 2)$ secreted by the hypothalamus may provide a new target for the research on the mechanism of obesity and therefore may contribute to the prevention of CAD. Schulze-Topphoff et al. (2009) provided evidence that Bdkrb1 may be a therapeutic target for chronic inflammation. TAS2R10 and TAS2R41 can stimulate the secretion of ghrelin in gastric fundic cells (Wang et al., 2019), yet they have not been studied in the cardiovascular system. These newly discovered genes in EAT that we have discovered may provide new targets for CAD research.

However, there are some limitations in our study. The main limitation of the study could be the ethical (quantity of total adipose tissue) limitation. Many CAD patients undergoing coronary artery bypass grafting have diabetes, which our study needs to exclude, making it even more difficult to obtain the samples. In addition, the present study stopped short of validating and further exploring the related mechanisms because of the limitation of culturing human epicardial adipocytes. Further studies are required to gain insight into the pathogenesis.

\section{REFERENCES}

Alipoor, E., Hosseinzadeh-Attar, M. J., Rezaei, M., Jazayeri, S., and Chapman, M. (2020). White adipose tissue browning in critical illness: A review of the evidence, mechanisms and future perspectives. Obes. Rev. 21:e13085.
In summary, in this study, we explored the gene expression profiles of EAT in CAD. We found that EAT may participate in CAD through key genes including GNG3, MCHR1, BDKRB1, MCHR2, CXCL8, CXCR5, CCR8, CCL4L1, TAS2R10, and $T A S 2 R 41$, and some novel pathways, including cytokine-cytokine receptor interaction, jak-STAT signaling pathway, nicotine addiction, chemokine signaling pathway, hematopoietic cell lineage, steroid hormone biosynthesis, butanoate metabolism, and neuroactive ligand-receptor interaction. These results may help us explore the role of EAT in CAD from a new and deeper perspective.

\section{DATA AVAILABILITY STATEMENT}

The datasets presented in this study can be found in online repositories. The names of the repository/repositories and accession number(s) can be found below: https://figshare.com/ and https://doi.org/10.6084/m9.figshare.12826109.v1.

\section{ETHICS STATEMENT}

The studies involving human participants were reviewed and approved by the Ethics Committee of Xiangya Hospital of Central South University. The patients/participants provided their written informed consent to participate in this study.

\section{AUTHOR CONTRIBUTIONS}

Q-CW and Z-YW designed the protocols and performed the experiments. QX and R-BL included the patients and collected the samples. G-GZ and R-ZS did the formal analysis. All the authors have contributed to the writing of the manuscript.

\section{FUNDING}

This work was supported by grants from the National Natural Science Foundation of China (Grant Nos. 81670267 and 81873479), the Outstanding Youth Foundation Project of Hunan Natural Science Foundation (Grant No. 2019JJ20036), and the Key R\&D Program of Hunan Provincial Department of Science and technology (Grant No. 2018SK2137).

\section{ACKNOWLEDGMENTS}

We thank Professor Wan-Jun Luo for his expert advice and helpful suggestions.

Balber, T., Benčurová, K., Kiefer, F. W., Kulterer, O. C., Klebermass, E. M., Egger, G., et al. (2019). In vitro Radiopharmaceutical Evidence for MCHR1 Binding Sites in Murine Brown Adipocytes. Front. Endocrinol. 10:324. doi: 10.3389/fendo.2019. 00324 
Bouchi, R., Terashima, M., Sasahara, Y., Asakawa, M., Fukuda, T., Takeuchi, T., et al. (2017). Luseogliflozin reduces epicardial fat accumulation in patients with type 2 diabetes: a pilot study. Cardiovasc. Diabetol. 16:32. doi: 10.1186/s12933017-0516-8

Chechi, K., Voisine, P., Mathieu, P., Laplante, M., Bonnet, S., Picard, F., et al. (2017). Functional characterization of the Ucpl-associated oxidative phenotype of human epicardial adipose tissue. Sci. Rep. 7:15566. doi: 10.1038/s41598-01715501- 7

Chong, C. R., Clarke, K., and Levelt, E. (2017). Metabolic Remodeling in Diabetic Cardiomyopathy. Cardiovasc. Res. 113, 422-430. doi: 10.1093/cvr/cvx018

Ding, R., Gao, W., He, Z., Wu, F., Chu, Y., Wu, J., et al. (2017). Circulating CD4(+)CXCR5(+) T cells contribute to proinflammatory responses in multiple ways in coronary artery disease. Int. Immunopharmacol. 52, 318-323. doi: 10. 1016/j.intimp.2017.09.028

Dyer, D. P., Thomson, J. M., Hermant, A., Jowitt, T. A., Handel, T. M., Proudfoot, A. E., et al. (2014). TSG-6 inhibits neutrophil migration via direct interaction with the chemokine CXCL8. J. Immunol. 192, 2177-2185. doi: 10.4049/ jimmunol.1300194

Frangogiannis, N. G. (2014). The inflammatory response in myocardial injury, repair, and remodelling. Nat. Rev. Cardiol. 11, 255-265. doi: 10.1038/nrcardio. 2014.28

Frangogiannis, N. G., and Entman, M. L. (2005). Chemokines in myocardial ischemia. Trends Cardiovasc. Med. 15, 163-169. doi: 10.1016/j.tcm.2005.06.005

Franssens, B. T., Nathoe, H. M., Leiner, T., van der Graaf, Y., and Visseren, F. L. (2017). Relation between cardiovascular disease risk factors and epicardial adipose tissue density on cardiac computed tomography in patients at high risk of cardiovascular events. Eur. J. Prev. Cardiol. 24, 660-670. doi: 10.1177/ 2047487316679524

Gäbel, G., Northoff, B. H., Weinzierl, I., Ludwig, S., Hinterseher, I., Wilfert, W., et al. (2017). Molecular Fingerprint for Terminal Abdominal Aortic Aneurysm Disease. J. Am. Heart Assoc. 6:6798. doi: 10.1161/jaha.117.006798

Gast, M., Rauch, B. H., Nakagawa, S., Haghikia, A., Jasina, A., Haas, J., et al. (2019). Immune system-mediated atherosclerosis caused by deficiency of long non-coding RNA MALAT1 in ApoE-/-mice. Cardiovasc. Res. 115, 302-314. doi: $10.1093 / \mathrm{cvr} / \mathrm{cvy} 202$

Gensini, G. F., Comeglio, M., and Colella, A. (1998). Classical risk factors and emerging elements in the risk profile for coronary artery disease. Eur. Heart J. 19(Suppl. A), A53-A61.

Gruzdeva, O., Uchasova, E., Dyleva, Y., Borodkina, D., Akbasheva, O., Antonova, L., et al. (2019). Adipocytes Directly Affect Coronary Artery Disease Pathogenesis via Induction of Adipokine and Cytokine Imbalances. Front. Immunol. 10:2163. doi: 10.3389/fimmu.2019.02163

Huang da, W., Sherman, B. T., and Lempicki, R. A. (2009). Systematic and integrative analysis of large gene lists using DAVID bioinformatics resources. Nat. Protoc. 4, 44-57. doi: 10.1038/nprot.2008.211

Iacobellis, G. (2015). Local and systemic effects of the multifaceted epicardial adipose tissue depot. Nat. Rev. Endocrinol. 11, 363-371. doi: 10.1038/nrendo. 2015.58

Iacobellis, G., Mohseni, M., Bianco, S. D., and Banga, P. K. (2017). Liraglutide causes large and rapid epicardial fat reduction. Obesity 25, 311-316. doi: 10. 1002/oby. 21718

Islam, S. A., Chang, D. S., Colvin, R. A., Byrne, M. H., McCully, M. L., Moser, B., et al. (2011). Mouse CCL8, a CCR8 agonist, promotes atopic dermatitis by recruiting IL-5+ T(H)2 cells. Nat. Immunol. 12, 167-177. doi: 10.1038/ni.1984

Kankaanpää, M., Lehto, H. R., Pärkkä, J. P., Komu, M., Viljanen, A., Ferrannini, E., et al. (2006). Myocardial triglyceride content and epicardial fat mass in human obesity: relationship to left ventricular function and serum free fatty acid levels. J. Clin. Endocrinol. Metab. 91, 4689-4695. doi: 10.1210/jc.2006-0584

Le Jemtel, T. H., Samson, R., Ayinapudi, K., Singh, T., and Oparil, S. (2019). Epicardial Adipose Tissue and Cardiovascular Disease. Curr. Hypertens. Rep. 21:36. doi: 10.1007/s11906-019-0939-6

Lin, Y. K., Chen, Y. C., Chang, S. L., Lin, Y. J., Chen, J. H., Yeh, Y. H., et al. (2013). Heart failure epicardial fat increases atrial arrhythmogenesis. Int. J. Cardiol. 167, 1979-1983. doi: 10.1016/j.ijcard.2012.05.009

Liu, Z., Wang, S., Wang, Y., Zhou, N., Shu, J., Stamm, C., et al. (2019). Association of epicardial adipose tissue attenuation with coronary atherosclerosis in patients with a high risk of coronary artery disease. Atherosclerosis 284, 230-236. doi: 10.1016/j.atherosclerosis.2019.01.033
Maghbooli, Z., and Hossein-Nezhad, A. (2015). Transcriptome and Molecular Endocrinology Aspects of Epicardial Adipose Tissue in Cardiovascular Diseases: A Systematic Review and Meta-Analysis of Observational Studies. Biomed. Res. Int. 2015:926567. doi: 10.1155/2015/926567

McKenney, M. L., Schultz, K. A., Boyd, J. H., Byrd, J. P., Alloosh, M., Teague, S. D., et al. (2014). Epicardial adipose excision slows the progression of porcine coronary atherosclerosis. J. Cardiothorac. Surg. 9:2. doi: 10.1186/1749-8090-9-2

Messerli, F. H., Hofstetter, L., Rimoldi, S. F., Rexhaj, E., and Bangalore, S. (2019). Risk Factor Variability and Cardiovascular Outcome: JACC Review Topic of the Week. J. Am. Coll. Cardiol. 73, 2596-2603. doi: 10.1016/j.jacc.2019.02.063

Milanese, G., Silva, M., Ledda, R. E., Goldoni, M., Nayak, S., Bruno, L., et al. (2020). Validity of epicardial fat volume as biomarker of coronary artery disease in symptomatic individuals: Results from the ALTER-BIO registry. Int. J. Cardiol. 314, 20-24. doi: 10.1016/j.ijcard.2020.04.031

Mráz, M., Cinkajzlová, A., Klouèková, J., Lacinová, Z., Kratochvílová, H., Lipš, M., et al. (2019). Dendritic Cells in Subcutaneous and Epicardial Adipose Tissue of Subjects with Type 2 Diabetes, Obesity, and Coronary Artery Disease. Mediat. Inflamm. 2019:5481725. doi: 10.1155/2019/5481725

Nappi, C., Ponsiglione, A., Acampa, W., Gaudieri, V., Zampella, E., Assante, R., et al. (2019). Relationship between epicardial adipose tissue and coronary vascular function in patients with suspected coronary artery disease and normal myocardial perfusion imaging. Eur. Heart J. Cardiovasc. Imaging 20, 13791387. doi: 10.1093/ehjci/jez182

Nomura, C. H., Assuncao-Jr, A. N., Guimarães, P. O., Liberato, G., Morais, T. C., Fahel, M. G., et al. (2020). Association between perivascular inflammation and downstream myocardial perfusion in patients with suspected coronary artery disease. Eur. Heart J. Cardiovasc. Imaging 21, 599-605. doi: 10.1093/ehjci/ jeaa023

Pezeshkian, M., and Mahtabipour, M. R. (2013). Epicardial and subcutaneous adipose tissue Fatty acids profiles in diabetic and non-diabetic patients candidate for coronary artery bypass graft. Bioimpacts 3, 83-89. doi: 10.5681/ bi.2013.004

Raggi, P., Gadiyaram, V., Zhang, C., Chen, Z., Lopaschuk, G., and Stillman, A. E. (2019). Statins Reduce Epicardial Adipose Tissue Attenuation Independent of Lipid Lowering: A Potential Pleiotropic Effect. J. Am. Heart Assoc. 8:e013104. doi: 10.1161/jaha.119.013104

Reinisch, I., Schreiber, R., and Prokesch, A. (2020). Regulation of thermogenic adipocytes during fasting and cold. Mol. Cell Endocrinol. 512:110869. doi: 10. 1016/j.mce.2020.110869

Schulze-Topphoff, U., Prat, A., Prozorovski, T., Siffrin, V., Paterka, M., Herz, J., et al. (2009). Activation of kinin receptor B1 limits encephalitogenic T lymphocyte recruitment to the central nervous system. Nat. Med. 15, 788-793. doi: $10.1038 / \mathrm{nm} .1980$

Schwindinger, W. F., Giger, K. E., Betz, K. S., Stauffer, A. M., Sunderlin, E. M., SimSelley, L. J., et al. (2004). Mice with deficiency of G protein gamma3 are lean and have seizures. Mol. Cell Biol. 24, 7758-7768. doi: 10.1128/mcb.24.17.77587768.2004

Sekimoto, R., Fukuda, S., Maeda, N., Tsushima, Y., Matsuda, K., Mori, T., et al. (2015). Visualized macrophage dynamics and significance of S100A8 in obese fat. Proc. Natl. Acad. Sci. U S A. 112, E2058-E2066. doi: 10.1073/pnas. 1409480112

Shannon, P., Markiel, A., Ozier, O., Baliga, N. S., Wang, J. T., Ramage, D., et al. (2003). Cytoscape: a software environment for integrated models of biomolecular interaction networks. Genome Res. 13, 2498-2504. doi: 10.1101/ gr.1239303

Szklarczyk, D., Gable, A. L., Lyon, D., Junge, A., Wyder, S., Huerta-Cepas, J., et al. (2019). STRING v11: protein-protein association networks with increased coverage, supporting functional discovery in genome-wide experimental datasets. Nucleic Acids Res. 47, D607-D613. doi: 10.1093/nar/gky1131

Vacca, M., Di Eusanio, M., Cariello, M., Graziano, G., D’Amore, S., Petridis, F. D., et al. (2016). Integrative miRNA and whole-genome analyses of epicardial adipose tissue in patients with coronary atherosclerosis. Cardiovasc. Res. 109, 228-239. doi: 10.1093/cvr/cvv266

Vianello, E., Dozio, E., Arnaboldi, F., Marazzi, M. G., Martinelli, C., Lamont, J., et al. (2016). Epicardial adipocyte hypertrophy: Association with M1polarization and toll-like receptor pathways in coronary artery disease patients. Nutr. Metab. Cardiovasc. Dis. 26, 246-253. doi: 10.1016/j.numecd.2015. 12.005 
Wang, Q., Liszt, K. I., Deloose, E., Canovai, E., Thijs, T., Farré, R., et al. (2019). Obesity alters adrenergic and chemosensory signaling pathways that regulate ghrelin secretion in the human gut. FASEB J. 33, 4907-4920.

Wong, C. X., Ganesan, A. N., and Selvanayagam, J. B. (2017). Epicardial fat and atrial fibrillation: current evidence, potential mechanisms, clinical implications, and future directions. Eur. Heart J. 38, 1294-1302. doi: 10.1093/eurheartj/ ehw045

Zengin, E., Bickel, C., Schnabel, R. B., Zeller, T., Lackner, K. J., Rupprecht, H. J., et al. (2015). Risk Factors of Coronary Artery Disease in Secondary PreventionResults from the AtheroGene-Study. PLoS One 10:e0131434. doi: 10.1371/ journal.pone.0131434
Conflict of Interest: The authors declare that the research was conducted in the absence of any commercial or financial relationships that could be construed as a potential conflict of interest.

Copyright () 2021 Wang, Wang, Xu, Li, Zhang and Shi. This is an open-access article distributed under the terms of the Creative Commons Attribution License (CC BY). The use, distribution or reproduction in other forums is permitted, provided the original author(s) and the copyright owner(s) are credited and that the original publication in this journal is cited, in accordance with accepted academic practice. No use, distribution or reproduction is permitted which does not comply with these terms. 